\title{
Experimental tests for foot pressure analysis during orthostatic position and gait
}

\author{
Daniel Ganea, Elena Mereuta, Silvia Veresiu, Madalina Rus, and Valentin Amortila* \\ "Dunarea de Jos" University of Galati, Department of Mechanical Engineering, 111 Domneasca \\ Street, Romania
}

\begin{abstract}
Human body postural deviation during normal activities such as gait or orthostatic position can cause injuries. The paper presents an experimental study regarding the distribution of contact pressures during gait and orthostatic position, aiming to evaluate the load distribution between forefoot $(\mathrm{FF})$ and rear-foot (RF), the projection of center of gravity and the support area variation while conducting stability metering and feet load distribution tests.
\end{abstract}

\section{Introduction}

The musculoskeletal system is a complex one and the asymmetry of motion stresses the human foot, which provides support and flexibility during weight transfer while standing or walking.

Posture ability is a neurological control activity dependence by nervous system that provides the optimal spatial framework for body adjustment. The input data for posture adjustment are received by the brain through various peripheral channels, processed and send to different systems for deployment. High plantar pressure generates posture abnormalities. Thus, an inadequate distribution on the plantar region occurs due to various factors such as eyesight deficiency, hearing deficiency, musculoskeletal system pathologies and mental states $[1,2]$.

Human body postural deviation during normal activities such as gait or orthostatic position can cause malfunctions or injuries not only to the foot but also in different areas of the body. Some studies highlight the fact that posture and equilibrium problems can be associated with rheumatology and orthopedic diseases such as back pain, neck tension and ankle instability [3]. Postural deviation is a state of imbalance that leads to functional overload, degeneration and dysfunction [1] and can be studied using photography, magnetic resonance imaging and $\mathrm{x}$-ray [4-7].

The anatomical structure which is basic for support and propulsion for any bipedal activity is the foot [8].

Due to its complex structure, the foot is responsible for maintain body balance in statics and dynamics, by symmetrical distribution of plantar pressure. Structurally, the human foot is divided in forefoot (FF), mid-foot, and the rear-foot (RF). Pressure distribution analysis for this anatomical structure implies sophisticate techniques such feet pressure distribution

* Corresponding co-author: valentin.amortila@ugal.ro 
and stability metering tests. Using these analyzing techniques, we can assess the malfunctions of the postural system by mapping the plantar pressure distribution.

The aim of this paper is to present an experimental study regarding the distribution of contact pressures during gait and orthostatic position, aiming to evaluate the load distribution between FF and RF, the projection of center of gravity and the support area variation while performing feet load distribution and stability metering tests.

\section{Method}

In this study, we conducted an analysis of foot pressure variation during standing and orthostatic position while performing feet load distribution and stability metering tests. All tests were performed in laboratory conditions without the influence of any external factors.

The feet pressure distribution test implies a static and a dynamic analysis. During the static test, the participant is asked to stand straight on the static area of the platform touching the specific alignment tool with the heel. The participant must be in a natural comfortable position. The software needs 5 seconds to determine the mean values of small oscillation. Using this method, the body weight distribution on the plantar surfaces and the geometry of both legs while standing straight are determined.

Dynamic analysis was used to determine the ground reaction evolution while walking. The participant performed a cycle of four completed steps equivalent with 12 footprints.

In order to measure the stability of a participant that stands a stability metering test was conducted. The test lasted 5 seconds while the participant stood with eyes closed.

For this experimental tests, we have used Diasu technologies. More exactly, the tests were performed by using a Podoscanalyzer and an UltraSensor LAC platform both combined with Milletrix Software capabilities.

\section{Results}

\subsection{Feet pressure distribution analysis}

\subsubsection{Static analysis}

The static test results indicates that the contact plantar surface is $182 \mathrm{~cm}^{2}$ (Fig. 1) with an average pressure of $450.5 \mathrm{~g} / \mathrm{cm}^{2}$. It can be noticed that the contact pressure is uneven distributed on both legs. Static analysis test revealed that the body center of pressure (CoP), corresponding with the body's gravity center (letter C in Fig. 2), is projected in the left of the support polygon. According to normal values, this deviation can indicate a moderate mono lateral deviation, with a hyper load. Thus, the left foot contact surface is $99.5 \mathrm{~cm}^{2}$ carrying $44.2 \mathrm{~kg}$ load, about $54 \%$ of body mass. The difference of $37.7 \mathrm{~kg}$ is distributed on the right foot on a smaller surface of $82.5 \mathrm{~cm}^{2}$.

In addition, it can be noticed that the projection of CoP is located towards back, indicating a more RF support, $64.20 \%$ for the left RF and $82.70 \%$ for the right RF. This was caused by the fact that the axis of the legs are not placed at the same angle. The right leg axis forms with the sagittal plane an angle of $26.2^{0}$, while the left leg an angle of $13.9^{0}$ (Fig. 3). Due to difference in positioning of both axis it can be seen that the center of gravity of the left foot (the letter $\mathrm{L}$ ) is placed toward the front of the foot to the right (point $\mathrm{R}$ ). In addition, this variation results to the fact that center of each foot $(\mathrm{CoF}$, letters $\mathrm{L}=$ left and $\mathrm{R}=$ right) reveals a misalignment with respect to $\mathrm{CoP}$. 
The maximum points of pressure (red colored) were detected on both rear feet, more on the left. These aspect have pointed out a mono lateral load. The maximum pressure of 1141 $\mathrm{g} / \mathrm{cm}^{2}$ (letter M) was detected in the right RF. The load distribution on each leg highlights an adequate bodyweight distribution between left and right legs, according to normal values (Fig. 4, 5). The load distribution between FF and RF for the left and right legs was comprised within the physiological values. The plantar surface was uneven distributed. Speaking of FF of both legs were highlighted an excessive surface's difference, more on the left. Between the $\mathrm{RF}$ a moderate difference of surface, more on the left was highlighted.

During static analysis, the foot geometry was divided in 6 areas: the FF (medial and lateral, including toes, if detected), the RF (medial and lateral) and the mid-foot (external/internal). The pressure distribution in this analysis, during orthostatic position was also uneven. It can be noticed that the RF of the human legs is more stressed as compared to FF. The mid-foot areas of both legs have almost no contact with the ground especially in the medial area.

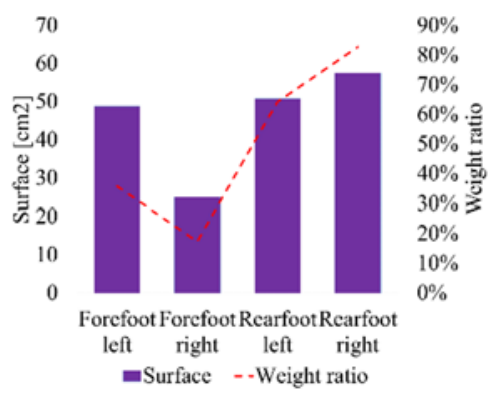

Fig. 1. Surface - weight ratio comparison for left foot and right foot division areas.

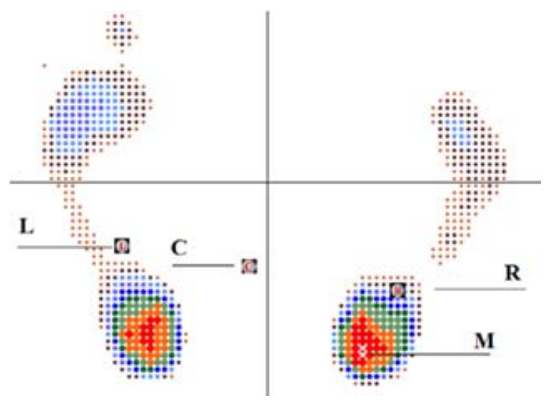

Fig. 2. Static analysis comparison between FF and $\mathrm{RF}$ divisions for left and right foot.

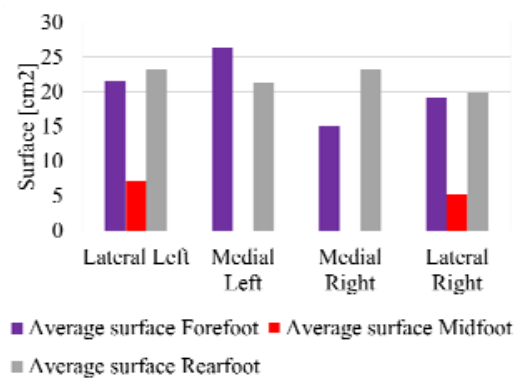

Fig. 4. Plantar average surfaces for medial and lateral areas.

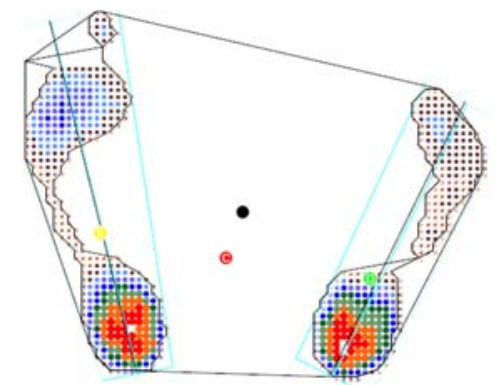

Fig. 3. The support polygon.

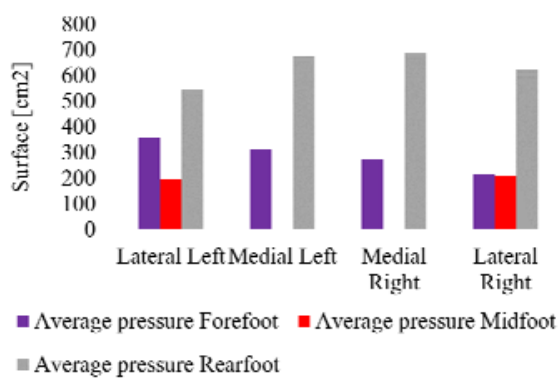

Fig. 5. Analysis of average static pressure. 


\subsubsection{Dynamic analysis}

During this test, the participant performed four completed steps equivalent with 12 footprints (Fig. 6).

The aim of this test was to analyze the ground reaction evolution within plantar region, during bipedal gait. The test was performed in laboratory conditions without the influence of any external factors, such as obstacles. Even so, the participant expressed a rather unstable gait generating an unstable posture.

By analyzing each image and dividing the footprint into FF, mid-foot and RF it can be noticed that the maximum point of pressure and the ground reaction varies from footprint to footprint. This is due to the fact that the geometry of each footprint varies.

During gait or standing, the plantar pressure should be distributed rather even and the maximum point of pressure should be applied within the RF surface.
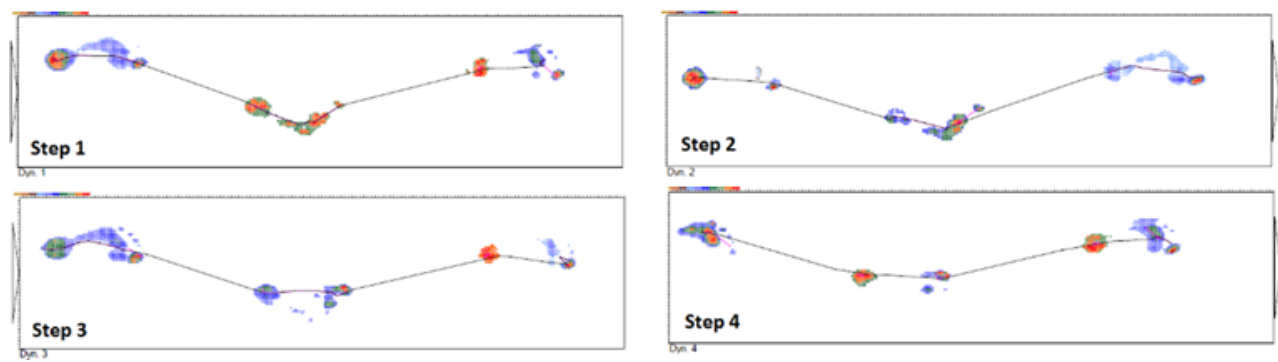

Fig. 6. Ground reaction evolution within plantar region, during bipedal gait.

By analyzing the data from the feet pressure distribution analysis, the data reveals an imbalance in the prevalence of weight load.

It can be noticed that during this test, even if the participant executed a controlled gait, the contact pressures and surfaces for both legs were variable (Fig. 7). By analyzing the gait cycle, the data reveals that both legs are stressed uneven.

By analyzing figure 7 we have noticed that during the dynamic test the maximum point of pressure for three of the footprints $(1,2$, and 9$)$ was smaller due to a more adequate plantar surface. Thus, a more efficient body mass distribution is emphasized. In this case, the plantar surface is ranging from $71.25 \mathrm{~cm}^{2}$ to $113.25 \mathrm{~cm}^{2}$. The body weight distribution generated a maximum point of pressure that varies from $1335.7 \mathrm{~g} / \mathrm{cm}^{2}$ to $1472 \mathrm{~g} / \mathrm{cm}^{2}$.

The average surface difference between FF and RF was $32 \%$ in the case of $18 \%$ loading.

In contrast are footprints number 4 and 12. The load is similar or greater that in the previous case and due to a very small plantar surface, $18 \mathrm{~cm}^{2}$, the maximum point of pressure exceeds $5000 \mathrm{~g} / \mathrm{cm}^{2}$.

By dividing the footprint in two areas, the RF and the FF, and by analyzing the mass distribution we can observe that not all surfaces are stressed in the same manner (Fig. 8). Normally, the body weight must be more concentrated in the heel area, known as the RF. This means that the heel joint is more in extension phase rather than flexion when approaching to ground. This pattern is notable for footprints number 2, 4, 5, 10 and 11. A particular case is footprint 6 . During this step an increased imbalance is noticeable. The maximum point of pressure is located within phalanges regions. More precisely, the foot approached the ground with a high heel angle of flexion rather than extension angle.

By analyzing figure 9, we have noticed that the left foot mean plantar surface, on average was twice as high as the right foot. For the left foot, it can be noticed that the full contact surface is completed in the middle of the step while for the right foot towards the end. 


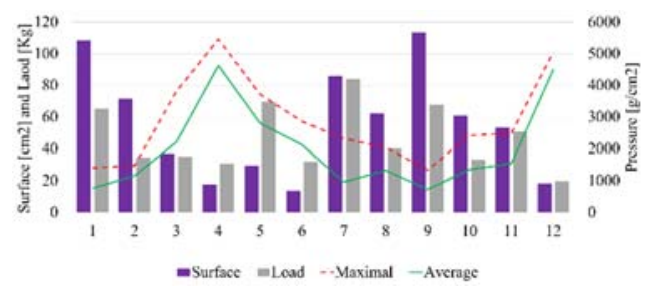

Fig. 7. Comparison between some dynamic analysis parameters for each of the 12 footprints.

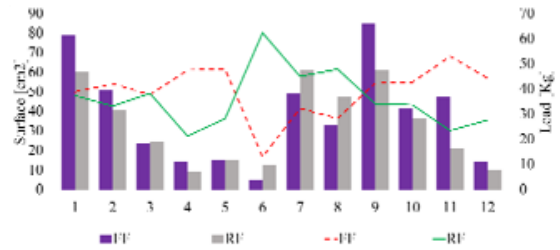

Fig. 8. A parallel between surface and load evolution while dividing each 12 footprints into $\mathrm{FF}$ and RF.

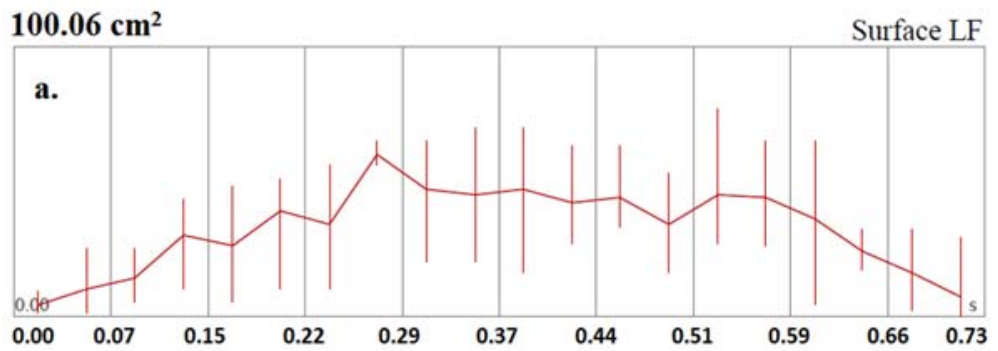

$100 \mathrm{~cm}^{2} \quad$ Surface RG

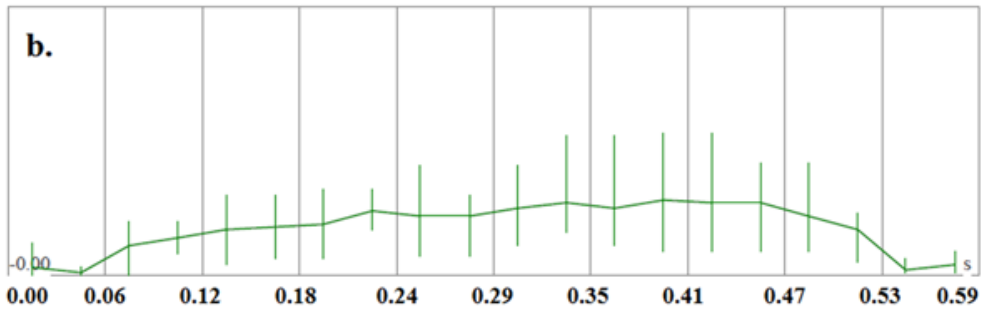

Fig. 9. Mean plantar surface; a. left foot; b. right foot.

\subsection{Stability metering}

The stability metering test objective was to study the body involuntary swing during standing. The test was performed in the absence of any external perturbations and any voluntary movements. Thus, the postural control system function such as steady - state can be analyzed by collecting information about the mean position of center of pressure $(\mathrm{CoP})$. Normally CoP displacement is within $1 \mathrm{~cm}^{2}$. The standing still position is inherently unstable due to small perturbation and fluctuations. By analyzing the data (Fig. 10), we have noticed that the participant posture has a small deviation towards left. This is noticeable because the support area is not equal divided on both legs. Thus the maximum point of pressure (red area) is located on the left RF. Due to a deviated towards left we noticed that the projection of CoP is shifted to left. The confidence ellipse (Fig. 11) with an area of $0.78 \mathrm{~cm}^{2}$ (major half axis of $0.85 \mathrm{~cm}$ and minor half axis of $0.29 \mathrm{~cm}$ ) and an eccentricity of 0.93 contains the projection of CoP during sway, described as the area that covers the center of a pattern with a given probability of $90 \%$. The ellipse major axis indicates the main direction of sway, $-31.1^{0}$.

The peaks of sway are represented in figure 12. By analyzing the radar balance graph we can easily evaluate the sway most important direction. The graph shows that body's involuntary swing direction while standing is oriented mainly towards right side of the body. 

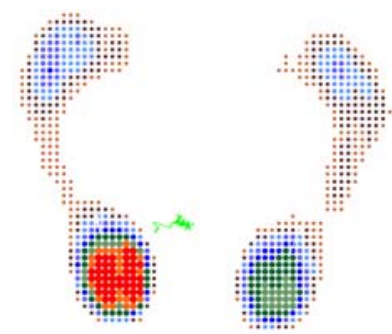

Fig. 10. The evolution of plantar stress while stability metering.

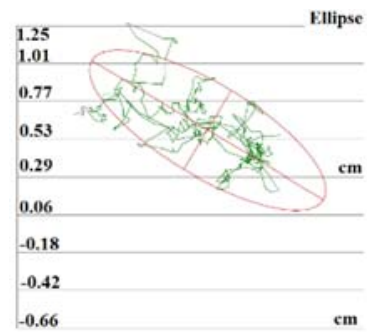

Fig. 11. The confidence ellipse.

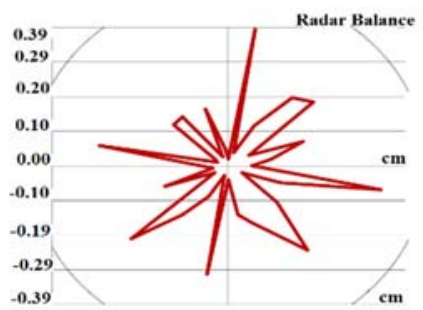

Fig. 12. Radar balance.

\section{Conclusions}

The foot is one of the most complex structure of the human body. By combining 26 bones connected with 33 joints and more than 100 muscle and tendons the human foot is a complex mechanical structure characterized by structural strength. Due to its complex geometry the human foot role is to absorb socks, to support the body weight offering balance and mobility.

Body posture is a neurological dependent ability. Also for an adequate posture, the human body needs a free of abnormalities mechanical structure. Thus, abnormalities that occurs in other parts of the body can influence the foot dynamics.

High plantar pressure may occur do to posture abnormalities. By using sophisticate technologies, we can evaluate the geometry and the dynamic of the plantar surfaces. By conducting stability metering and feet pressure distribution analysis, we can evaluate the area that usually receive the maximum pressure. Anatomical speaking, these areas are the heel and metatarsal head.

By analyzing pressure and plantar surfaces, we can conclude that the participant has no abnormalities like flat foot or high arch and hyperextension of the toes. The high arch and hyperextension of the toes can be indicated by high pressure associated with a decreased support within the midfoot area. On the other hand, flat foot represents low stress with an increased plantar surface. In our study, the data indicates that the participant does not present any of these abnormalities. However, tests like feet pressure distribution and stability metering have an excellent potential to provide top research in the field of human body posture.

\section{References}

1. M. Bellizzi, R. Rizzo, G. Bellizzi, M. Ranieri, M. Fanelli, G. Megna, U. Procoli, Strabismus.19, 1,(2011)

2. B. Bricot, Total Postural Reprogramming (Dux Lucis Books, Santa Monica, 2008)

3. B. Missaoui, P. Portero, S. Bendaya, O. Hanktie, P. Thoumie, Clin. Neurophysiol.38, 6 (2008)

4. J.L.P. Rosário, I.Y.Nakashima, K. Rizopoulos, D. Kostopoulos, A.P. Marques, Clinical Chiropractic.15, 3-4 (2012)

5. J.S. Steffen, I. Obeid, N. Aurouer, O. Hauger, J.M. Vital, J. Dubousset, W. Skalli, European Spine Journal.19, 5 (2010)

6. E. Berthonnaud, J. Dimnet, R. Hilmi, Comput. Med. Imaging Graphics.33, 8 (2009)

7. H. Suzuki, K. Endo, J. Mizuochi, H. Kobayashi, H. Tanaka, K. Yamamoto, Eur. Spine J. 19 (2010)

8. J.L. Rosário, J Bodyw, Mov Ther.18, 2 (2014) 